\title{
Study on Registration Algorithm of Section Profile of Automobile Sealing Strip Based on Angle Histogram
}

\author{
Zhongliang Meng ${ }^{1, a}$, Lei He $\mathrm{H}^{2, b}$ \\ ${ }^{1}$ School of Mechanical Engineering, Zaozhuang University, Zaozhuang 277160, P. R. China \\ ${ }^{2}$ School of Mechanical Engineering, Guizhou University, Guiyang 550025, P. R. China \\ ameng198512@126.com, ${ }^{\mathrm{b}}$ 52773057@qq.com
}

Keywords: Automobile sealing strip, section profile, precise registration, detection.

\begin{abstract}
A registration algorithm is proposed based on angle histogram in respect of the inspection of section profile of automobile sealing strip. Firstly, the edge data of image contour will be extracted, on the basis of which the convex hull of contour will be used. Then through the convex hull, the position and angle parameter of the rectangle with the minimum surrounding area will be obtained to complete the coarse registration, on the basis of which, the precise registration is achieved through angle histogram at last.
\end{abstract}

\section{Introduction}

Currently, the shape of section profile of sealing strip is still detected by hand in lots of sealing strip manufacturers in China. Repeated detection should be conducted constantly in the whole process of production. In case of defective goods, strips at least $50 \mathrm{~m}$ long will be abandoned later. Besides, they are hard to control for unstable detection time, and flow into the next procedure often. Therefore, it is of very importance to detect the product quality online and automatically in the production process of sealing strips. In the defect inspection, the section of sealing strip to be tested will be compared with that of standard sealing strip in order to find out the differences. However, the position and angle of the section of sealing strip are different, thus the image registration needs to be used to "align" the spatial position between the sealing strip to be tested and standard sealing strip in order to lay a foundation for the next defect judgment. In the machine vision, if a piece of image with certain specific target is given, the position of target should be found from another one with certain specific target and two goals need to be aligned in the space, hence the image registration is used.

\section{Edge extraction}

In the image processing, such features of the target as contour and shape are very importance characteristic parameters. Images obtained from the previous edge detection are just some edge symbols. Before quantitative characteristic analysis and description, the following information needs to be aware: coordinate of each of edge pixel point, its belongings, and the pixel point before and after, how many pixel points in the edge, all of which are in need of edge extraction. Pursuant to the scanning order, there is not the edge point above and on the left of the first point. Therefore, eight neighborhood points are inspected anticlockwise from the bottom left. Similarly, the next edge point will be found in the order of detection and according to the characteristics of eight neighborhoods and then the next edge point after next will be inspected continuously clockwise from the next edge point until returning to the original point or failing to detect the next point. The whole extraction process is shown in Fig. 1. 


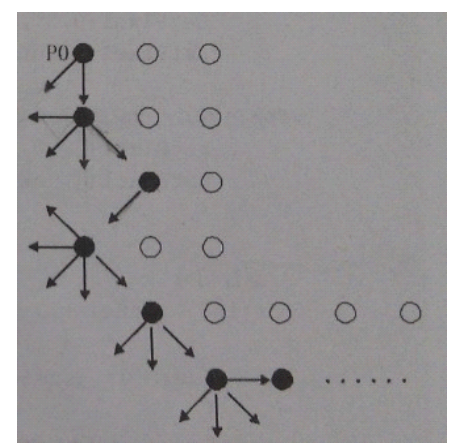

Fig.1 Sketch map of contour extraction

\section{Convex Hull}

For the calculation of the rectangle with the minimum surrounding area in the target area, the convex hull shall be calculated firstly. In a certain area, the convex hull of a point set is the minimum convex set of all the points within the area. If all the points connected by any two points in a point set belong to this point set, it is a convex hull, as is shown in Fig. 2.

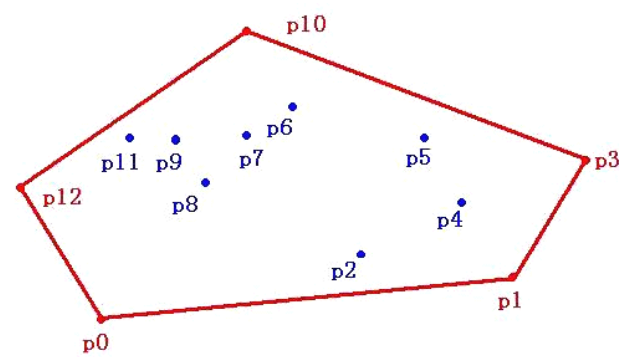

Fig.2 Convex Hull

The convex hull of polygon $P$ refers to that with the minimum area surrounding P. Informally, if a rubber band surrounds a polygon $\mathrm{P}$, the shape when the rubber band is tight is the convex hull of $\mathrm{P}$. The convex hull of standard image and two images to be tested in different positions is shown in Fig.3.
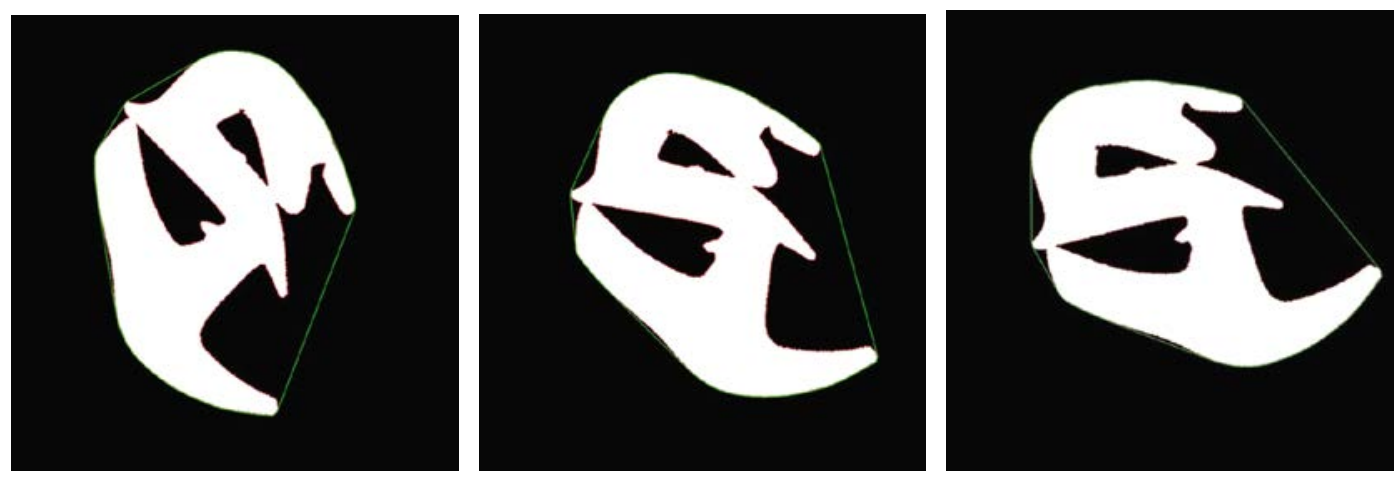

(a)Convex hull of standard image, (b) Convex hull of the image 1 to be tested, (c) Convex hull of the image 2 to be tested

Fig.3 Convex hull of standard image and image to be tested

\section{Rectangle with the minimum surrounding area}

The rectangle with the minimum surrounding area of the section of sealing strips in different directions is shown in Fig. 4. 

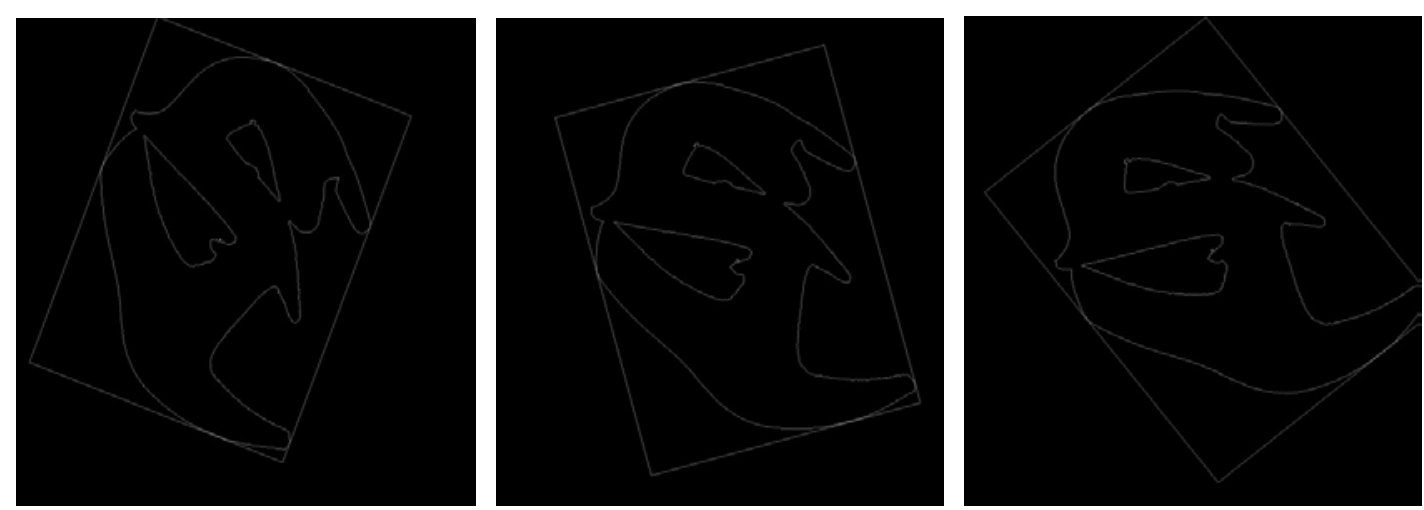

(a)Rectangle with the minimum surrounding area of the standard image, (b) Rectangle with the minimum surrounding area of the image 1 to be tested, (c) Rectangle with the minimum surrounding area of the image 2 to be tested

Fig.4 Rectangle with the minimum surrounding area of the standard image and image to be tested

It can be seen from calculation, the parameter of the rectangle with the minimum surrounding area are little linked to most of the edge fragments of the section contour, but more linked to the points connecting rectangle and contour and points surrounding the edge points. As a result, the registering which is achieved by way of calculating the rectangle with the minimum surrounding area is the coarse registration with possible error, and the characteristic parameter needs to be extracted further for precise registration.

It is found from analysis that the error magnitude between the image to be tested and standard image is positive to the extent to which these critical edge points deform and distort. The point, curve and regional characteristic based characteristic registration cannot be extracted stably because the outline of automobile sealing strip can deform at any position. To solve this problem, the registration algorithm based on the angle histogram is proposed for precise registration. The length of the long and short axle of rectangle with the minimum surrounding area represents the whole shape of contour edge, and the maximum and minimum length of long and short axles can be set as threshold through the comparison of tolerance of sections of different dimensions. Only when the long and short axles meet required length, the precise registration is needed. If not, it is judged to be defective.

\section{Angle histogram}

The horizontal ordinate of the angle histogram stands for the angle while the vertical ordinate stands for the number of every angle. In calculation, the value from 360 degrees should be added to 0 degree. The angle histogram of different images, as is shown in Fig. 5, is the angle histogram of standard sealing strip image and those sealing strip images in other positions. It can be seen that they are generally similar in shape, in other words, when their angle histogram is the most similar, the angle difference and position difference are the final moving distance and rotation angle through constant fine adjustment and comparison of the angle histogram in the new position. After abandoning the large deformation through the previous threshold, the small deformation will still influence the angle and central position of the rectangle with the minimum surrounding area, but the angle and central position will falls in the same scope.

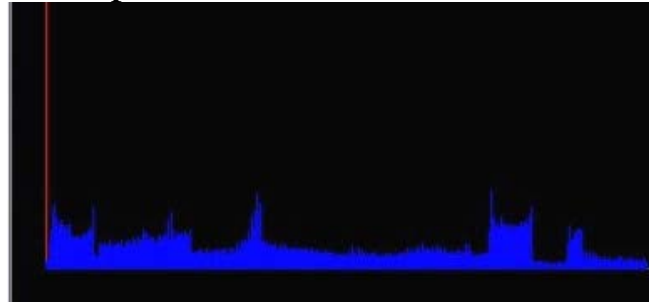

(a)Angle histogram of standard image,

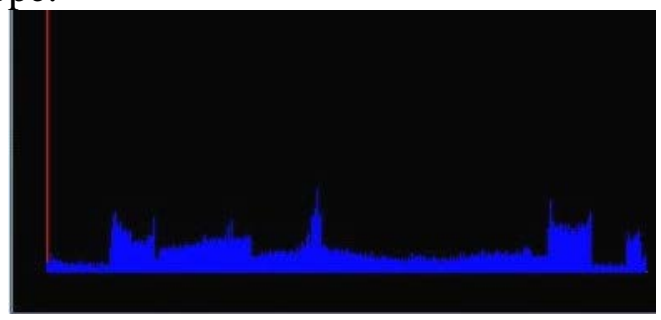

(b) Angle histogram of image 1 to be tested 


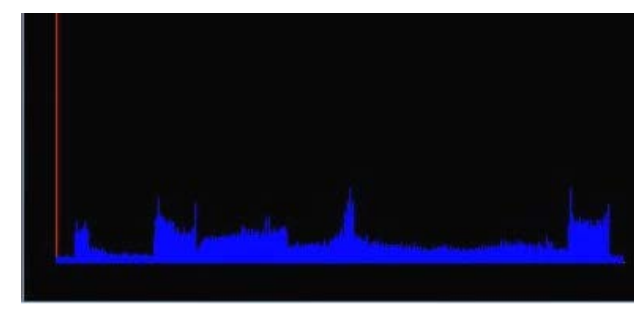

(c)Angle histogram of image 2 to be tested

Fig.5 Angle histogram of standard image and image to be tested

After several tests, an area will be set as the searching area of the center surrounding the central position of the rectangle with the minimum surrounding area; and an area ranging from -5 degrees to 5 degrees will be set as a searching area of the angle surrounding the deviation angle as follows: set some point of the central searching area as a center and move it to the center of standard image. Then rotate the image to be tested within the scope of initial deviation angle ranging from -5 to 5 degrees and calculate the angle, thus getting "angle histogram" under the center. The angle histogram between the image to be tested and standard image is compared by way of Bhattacharyya. Then, the position with minimum result is regarded as the best matching position of the angle histogram (by way of Bhattacharyya, ranging from 0 to 1: 0 is completely matching while 1 is completely mismatching.) The moving distance and angle difference are regarded as the final stiffness parameter.

The images corresponding to the histogram above will be registered based on the algorithm in this chapter as is shown in Fig. 6. Image b is a part of image a while image $d$ is a part of image c. In these four images, white color stands for the edge of standard image, light gray stands for the edge of image to be tested. In actual practice, after stiffness exchange, the image to be tested will be sampled and shown in the standard image. If the standard image and image to be tested are edges in some pixel point, the data of image to be tested will replace the data of the standard image.
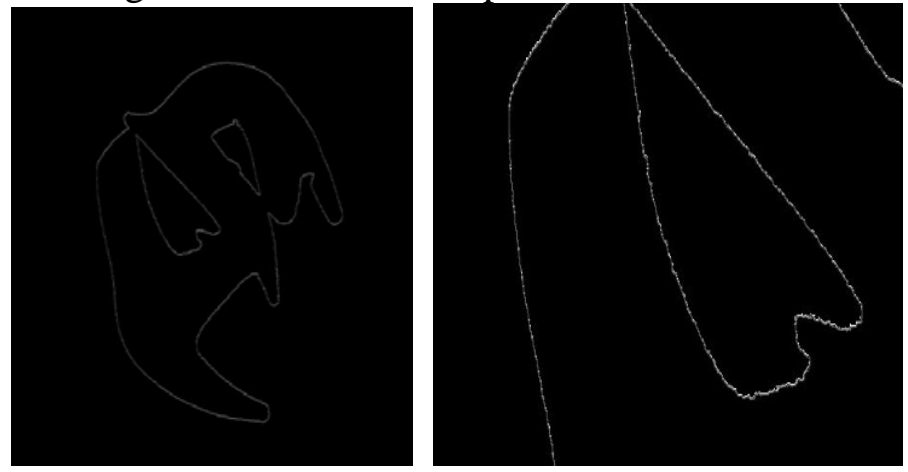

(a)1 test image and the standard image overlays (b)Figure (a) partial view

It can be seen from the figure that most of the edges are light gray, which shows that the registered tested image overlaps with the standard image highly.

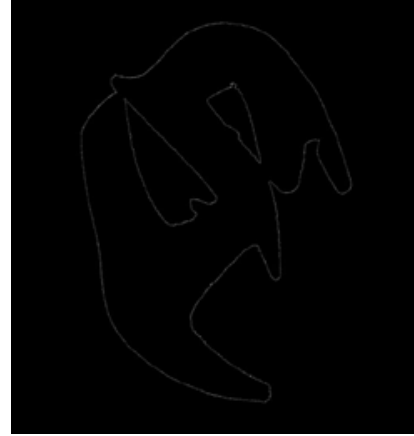

(c)2 test image and the standard image overlays

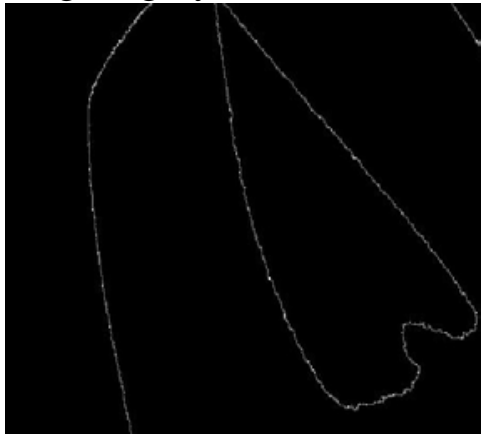

(d)Figure (c) partial view

Fig.6 Overlap image and partial image between two images to be tested and standard image 


\section{Summary}

Partial characteristics, such as angle point, curve rate, are not allowed to be used, because the section profile of sealing strip will deform in any position. In the whole process of registration, the calculation characteristic is the whole section profile of the sealing strip. Before registration, the edge of target should be extracted in the image, and such information as coordinate and subordinated edge for lower edge point is recorded. It is divided into coarse registration and precise registration. According to the characteristics of section deformation, the precise registration method is proposed based on angle histogram and the searching space is set within the scope of rectangle center and angle ranging from -5 to 5 degrees, and the step is a pixel point and 1 degree. Extrinsic cycle is conducted based on the movement of rectangle center and the intrinsic cycle is conducted based on the movement of angle. Then the angle from the ling segment from every edge point to rectangle center point to positive direction of axle $\mathrm{X}$ is calculated and accounted to make an angle histogram. With angle histogram being a similarity measurement and the angle and central difference of the optimal similarity measurement being the parameter of the final stiffness exchange, the precise registration is completed to improve the detection precision of automobile sealing strip.

\section{References}

[1] Barbara Zitova,Jan Flusser.Image registration methods:a survey[C].Imaging and Vision Computing.2003(11),p. 977-1000.

[2] Wang Hongmei,Zhang Ke,Li Yanjun.Image Matching Research and Development[J]. Computer Engineering and Applications,2004, 19.p. 42-46..

[3] Rafael C.Donzalez,Richard E.Woods.Digital image processing (third edition) [M].Beijing: Electronic Industry Press, 2012. p. 98-112.

[4] Avraham A.Melkman.On-line construction of the convex hull of a simple polyline[C]. Information Processing Letters, 1987( 25).p.11-12.

[5] H. Freeman,R.Shapira.Determining the minimum-area encasing rectangle for an arbitrary closed curve[J]. Communications.Vol.18(1975) No.11,p.409-413.

[6] G.Toussaint. Solving geometric problems with the rotating calipers[C]. Proceedings of IEEE MELECON', 1983, p. 131-136. 